\title{
ORIGINAL
}

\section{FACTORES DE RIESGO PARA LA ENFERMEDAD TUBERCULOSA EN LOS CASOS DE SIDA NOTIFICADOS EN BRASIL, 1980 A 2000}

\author{
Josué Laguardia (1), Edgar Merchán-Hamann (2)
}

(1) Escola Nacional de Saúde Pública - Fundação Oswaldo Cruz. Rio de Janeiro, RJ, Brasil.

(2) Departamento de Saúde Coletiva - Faculdade de Ciências da Saúde - Universidade de Brasília. Brasilia, DF, Brasil.

\section{RESUMEN}

Fundamento: El sida afecta las características epidemiológicas de la tuberculosis, tanto por cambios en su progresión clínica en los enfermos coinfectados, como por el incremento del número de casos de tuberculosis en personas VIH positivos/sida. Esto puede llevar a un aumento de la transmisión del bacilo en la población. El objetivo de este trabajo es identificar los factores asociados a la ocurrencia de tuberculosis en casos de sida notificados al Ministerio de la Salud del Brasil.

Metodos: Se realizó un estudio epidemiológico transversal, siendo los casos de sida clasificados inicialmente en 2 categorías: (1) con tuberculosis y (2) sin tuberculosis. Posteriormente fueron comparados 4 grupos : (1) sin infección oportunista; (2) sólo con tuberculosis (pulmonar y/o diseminada); (3) con otras infección oportunista excepto tuberculosis, y (4) con tuberculosis asociada a otras infecciónes oportunistas. El análisis bivariado fue ajustado por regresión logística.

Resultados: En el análisis de regresión, las prevalencias más altas de tuberculosis estaban asociadas significativamente a edad menor de 40 años, baja escolaridad, residencia en la región sureste del país y diagnóstico efectuado antes de 1996. La forma de transmisión más asociada con la presencia de tuberculosis fue el uso de droga inyectable, seguida de las relaciones heterosexuales. Al comparar 4 períodos consecutivos de evolución de la definición de caso de sida para notificación hubo disminución de casos con tuberculosis desde 1996, así como aumentos significativos en la proporción de pacientes sin infección oportunista.

Conclusiones: Sexo, edad y escolaridad son predictores de la presencia de tuberculosis entre las infecciónes oportunistas.

Palabras clave: Síndrome de inmunodeficiencia adquirida. Tuberculosis. Prevalencia. Factores de riesgo.

\section{ABSTRACT}

Risk Factors for Tuberculosis in the AIDS Cases Reported in Brazil, 1980-2000

Background: Aids affects the epidemiological characteristics of tuberculosis, due to both the changes in the clinical progress of the jointly affected patients, as well as the increase in the number of jointly affected patients, as well as the increase in the number of
tuberculosis cases among individuals having tested positive for tuberculosis cases among individuals having tested positive for
hiv/aids. This may lead to an increase in the transmission of the bacillus among the population. this study is aimed at identifying the factors related to the occurrence of tuberculosis among aids cases reported to the brazilian ministry of health.

Methods: a cross-sectional epidemiological study was conducted, the aids cases initially having been classified into 2 categories (1) with tuberculosis and (2) without tuberculosis. a comparison was subsequently drawn among 4 groups: (1) no opportunist infection, (2) only tuberculosis (pulmonary and/or disseminated), (3) with other opportunist infections except tuberculosis and (4) with tuberculosis in conjunction with other opportunist infections. The bivariaculosis in conjunction with other opportunist inf
te analysis was adjusted by logistic regression.

Results: in the regression analysis, the highest tuberculosis prevalences were significantly linked to age under 40 , little schooling, living in the southeast region of the country and diagnosis made prior to 1996 . The means of transmission most closely associated to the presence of tuberculosis was the use of intravenous drugs, followed by heterosexual relations. when 4 consecutive periods in the evolution of the reporting aids case definition, there had been a decrease in cases with tuberculosis since 1996, as well as significant increases in the proportion of patients with no opportunist infection.

Conclusions: Gender, age and school attendance are predictors of the presence of tuberculosis among opportunist infections.

Keywords: AIDS. Tuberculosis. Cross-sectional study. Risk factors.

\section{INTRODUCCIÓN}

Correspondencia:

Josué Laguardia.

Rua do Russel, 404, Apartamento 504.

Gloria, Rio de Janeiro, RJ

CEP : 22.210-010. Brasil.

Tel. 55-21-2285-1809

Correo electrónico: josue_laguardia@yahoo.com.br
Desde el surgimiento del sida, la tuberculosis y la infección por el Virus de la Inmunodeficiencia Humana (HIV) han estado relacionados. La inmunosupresión resultan- 
te del sida tiene un efecto directo e indirecto en las características epidemiológicas de la tuberculosis; hay cambios en la progresión clínica de la tuberculosis en pacientes coinfectados por VIH y Mycobacterium tuberculosis, asi como un incremento en el número de casos de tuberculosis en pacientes VIH positivos/sida colaborando para un aumento de la transmisión de la infección por bacilo de la tuberculosis en la población general ${ }^{1,2}$. En 1987, la revisión de los criterios de definición de caso de sida para efectos de vigilancia epidemiológica, realizada por los Centers for Disease Control and Prevention $^{3}$, incluyó la tuberculosis extrapulmonar como «enfermedad indicadora». Además de los criterios de comprobación laboratorial, tal inclusión presupone que es más probable la presencia de inmunosupresión avanzada cuando ella no está confinada al pulmón ${ }^{4}$.

En el Brasil, la revisión oficial de la definición de caso de sida en adultos, efectuada en 1992, tuvo por objetivo aumentar la sensibilidad de la definición previa ${ }^{5,6}$. La tuberculosis diseminada, extrapulmonar y/o pulmonar no cavitaria fueron incluídas como entidades que por si solas definen un caso de sida. Por outra parte, la tuberculosis pulmonar cavitaria o la no especificada necesitan otros criterios para la definición de caso.

En los países en desarrollo, la tuberculosis figura entre las tres principais enfermedades oportunistas en pacientes seropositivos para HIV siendo que hay mayores tasas de incidencia de tuberculosis y mayor riesgo de desarrollar la enfermedad en las personas seropositivas para el $\mathrm{VIH}^{7-11}$. Datos de los paises desarrollados ${ }^{12,13}$, mostraron que el riesgo de progresión para tuberculosis activa entre individuos que utilizan drogas y están infectados por el HIV es mucho mayor que en individuos seronegativos. En Londres, un estudio caso-control anidado en una cohorte retrospectiva de pacientes positivos para el virus VIH-1 determinó que los factores associados a la tuberculosis fueron el origen africano sub-saharino y la edad menor que 28 años de los participantes $^{14}$.

Aunque aún no esté suficientemente claro si la fuente de infección para la tuberculosis asociada con HIV es usualmente una infección primaria reactivada o una infección exógena secundaria, se sabe que las personas infectadas con HIV y M. tuberculosis tienen un riesgo anual de progresión de 5 a $15 \%{ }^{15}$. La introducción de la terapia antiretroviral altamente activa (HAART) ha producido cambios es estos niveles de riesgo causando una disminución de la mortalidad e incidencia de las infecciones oportunistas alrededor de $60 \%$ a $90 \%$ en las personas viviendo con sida en paises desarrolados ${ }^{16}$.

En el periodo de enero de 1980 a junio de 2001, fueron notificados en el Brasil 215.805 casos de $\operatorname{sida}^{17}$ y hasta 1999 , la tuberculosis era la tercera principal enfermedad oportunista, en orden de importancia, acometiendo cerca de $18 \%$ de los pacientes con sida notificados en el Brasil. Se estima que 17,7\% de los casos de tuberculosis en el Brasil son debidos a la infección por el HIV ${ }^{18}$.

Estudios efectuados entre 1987 y 1991 en el Brasil, en pacientes con tuberculosis atendidos en centros para tratamiento de esta enfermedad, mostraron niveles de seroprevalencia para HIV que variaban de 0,5 a $20,7 \% 19,20,21,22$. Tales prevalencias, de un modo general, fueron mayores en portadores de formas extrapulmonares, siendo la ganglionar la más frecuente, en individuos de sexo masculino, en la edad adulta (20-59 años), con menor nivel educacional y con relato de prácticas heterosexuales ${ }^{19,23,24,25}$. La tuberculosis estuvo presente como causa associada en cerca de $20 \%$ de las 4.619 defunciones por sida ocurridas en $1998^{26}$.

En la última década, el gobierno brasileño ha implementado una red de laboratorios que realizan la prueba para detección del VIH. No obstante, la articulación de ese sistema con otras instancias de los servicios de 
salud es muy heterogénea y el acceso a la prueba no se encuentra disponible, en la práctica, en todos los lugares donde la tuberculosis es diagnosticada y tratada. En el Brasil, se detectan entre 80.000 y 90.000 casos de tuberculosis por año, siendo que el coeficiente de incidencia es de cerca de 50 casos por 100.000 habitantes. Los indicadores de desempeño del programa de control de la tuberculosis están lejos de ser satisfactorios; em 1999, en 29.707 casos cuyo desenlace fue cuidadosamente verificado, la curación fue obtenida en $8,8 \% ; 12,7 \%$ abandonaron el tratamiento y $63,3 \%$ lo completaron. La letalidad fue de $5,9 \%{ }^{27,28}$. Uno de los factores que más afecta el adecuado control de la tuberculosis está relacionado con la calidad de los servicios de salud, pues el acceso a ellos y su organización son precarios. La sospecha diagnóstica y confirmación de tuberculosis también es limitada y relativamente tardía.

La iniciativa de DOTS, llamada en el Brasil Tratamento Diretamente Observado (TDO), tendiente a mejorar el desempeño terapéutico y el control de la tuberculosis, fue incluída como parte del Programa Nacional de Control de la Tuberculosis en 1998, pero todavía no es una realidad consolidada en la mayor parte del país. El gobierno brasileño ha logrado que el tratamiento para VIH-sida esté disponible para casi todos los pacientes ${ }^{29}$. Sin embargo, debido a la falta de integración de los servicios, la mayoría de los profesionales aún no ha sido sensibilizada para la situación impuesta por el advenimiento de la coinfección ${ }^{30}$. Datos del mayor municipio del país, São Paulo, indican que $22 \%$ de las muertes por tuberculosis son atribuibles a la coinfección con el VIH y que la falta de asociación entre la mortalidad por tuberculosis y la utilización de los servicios de salud sugiere una reducida efectividad del programa de control $^{31}$. Varios estudios destacan la importancia de la articulación de los servicios en el contexto de la coinfección, lo que cobra mayor relevancia en los países en desarrollo ${ }^{32,33}$.
El presente estudio tuvo en vista la necesidad de orientar los profesionales de salud hacia la sospecha y comprobación del diagnóstico de infección por el HIV en pacientes con tuberculosis atendidos en los servicios públicos de salud. Su objetivo fue investigar los factores asociados a la ocurrencia de la tuberculosis como infección oportunista en el momento del diagnóstico y notificación de los casos de sida.

\section{MATERIAL Y MÉTODOS}

Fue realizado un estudio epidemiológico analítico transversal para definir las variables predictoras de la tuberculosis entre los individuos con sida notificados de 1980 a marzo de 2000. Fueron seleccionados todos los casos notificados de sida que a su vez habían sido confirmados previamente y que ya satisfacían los criterios oficiales estandarizados de definición de caso vigentes. Tales informaciones son procesadas por los programas de control de la enfermedad de los estados brasileños y posteriormente enviadas a la Coordinación Nacional de Enfermedades Sexualmente Transmisibles y sida del Ministerio de la Salud en Brasilia (CNDST/AIDS). Se utiliza un sistema de notificación computadorizado (Sistema de Informação Nacional de Agravos de Notificação - SINAN) que no acepta el registro, en la base de datos, de los casos que no llenan los critérios de definición de caso.

Para el análisis de los datos, inicialmente fueron comparados dos grandes grupos de casos: (1) los que tenían tuberculosis como diagnóstico en el momento de la notificación, y (2) los que no la tenían. El análisis de posibles asociaciones con otros factores fue realizado de manera separada para hombres y mujeres. En el análisis bivariado, fueron calculadas las razones de prevalencia y los respectivos intervalos de confianza de $95 \%$. Las asociaciones fueron probadas utilizando el Chi cuadrado para variables 
dicotómicas así como pruebas de diferencias de promedios y medianas para variables continuas. El análisis multivariado fue realizado por medio de la regresión logística binaria, para estimar los chances (o Odds) de presentar tuberculosis. Después de ser definida como variable resultante la presencia de tuberculosis, fueron incorporadas al modelo las variables dicotomizadas más relevantes de acuerdo con el análisis bivariado. El análisis estadístico fue efectuado utilizando los programas EPIINFO 6.04 y SPSS $9.0^{34,35}$.

Un análisis más detallado fue realizado agrupando los pacientes en cuatro categorías según la presencia y tipo de infección oportunista: (1) sin infección oportunista; (2) sólo con tuberculosis (pulmonar y/o diseminada); (3) con otras infecciones oportunistas excepto tuberculosis, y (4), con tuberculosis ocurriendo concomitantemente con otras infecciones oportunistas. El análisis bivariado fue realizado de la misma manera que fue descrito. Un nuevo análisis multivariado fue realizado utilizando la misma técnica, con el objetivo de estimar los chances (o Odds) de presentar tuberculosis aislada o asociada a otras infecciones oportunistas utilizando como grupo de comparación los casos sin infección oportunista. Después de ser definida como variable resultante la categoría según la presencia y tipo de infección oportunista, fueron incorporadas al modelo las variables dicotomizadas más relevantes de acuerdo con el análisis bivariado.

Las categorías de exposición y las otras variables fueron utilizadas según las informaciones contenidas en la ficha de investigación de sida. Para la comparación de las tendencias a lo largo del periodo estudiado, los periodos fueron determinados tomando en cuenta las revisiones de las definiciones de caso de sida, con la inclusión de tuberculosis diseminada o extrapulmonar (1987) y posteriormente pulmonar (1992), así como la introducción de la terapia triple antiretroviral (1996).

\section{RESULTADOS}

El análisis descriptivo de los 184.506 casos de sida notificados entre 1980 y marzo de 2000 reveló que $75 \%$ eran de sexo masculino, con edades promedio de 33,1 años $(\mathrm{DS}=11,2$; mediana $=32)$. Con respecto al nivel de estudios, $60 \%$ habían cursado hasta el $4^{\circ}$ año de la educación básica. La categoría de transmisión predominante fue el contagio por medio de relaciones heterosexuales (32\% del total de los casos). La región de residencia de $70 \%$ de los casos correspondía el sureste del Brasil, que comprende los estados de São Paulo, Rio de Janeiro, Minas Gerais y Espírito Santo.

Con respecto a la presencia de tuberculosis en el momento de la notificación, cerca de $24 \%$ de los casos notificados de sida tenían ese diagnóstico. La tabla 1 muestra la distribución de frecuencias según algunas variables presentes en la ficha de notificación. Entre los casos con tuberculosis había una proporción significativamente mayor de individuos pertenecientes al sexo masculino, de baja escolaridad, de casos cuyo mecanismo de transmisión fue el uso de drogas inyectables y de notificaciones originadas en la región Sureste del país entre 1987 y 1995. Los casos con tuberculosis tuvieron un promedio de edad un poco mayor siendo éste significativo.

El análisis bivariado y ajustado por regresión en casos ocurridos entre individuos de sexo masculino (Tabla 2) comprobó que mayores prevalencias de tuberculosis estaban asociadas significativamente a los siguientes factores, en orden de importancia: transmisión por uso de drogas inyectables, herosexual y homo-bisexual; baja escolaridad, residencia en la región sureste del país, diagnóstico efectuado antes de 1996 y edades menores a 40 años. Las categorías de transmisión por medio de relaciones homosexuales y bisexuales fueron comparados con las restantes y se observó en el análisis bivariado que se comportaban como factores 
Tabla 1

Distribución de los casos notificados de sida según la presencia de tuberculosis (TB) de acuerdo con variables seleccionadas. Brasil, 1980-2000

\begin{tabular}{|c|c|c|c|c|c|}
\hline \multirow[b]{2}{*}{ Variables } & \multicolumn{2}{|r|}{ Sin TB } & \multicolumn{2}{|r|}{ Con TB } & \multirow[b]{2}{*}{$\mathrm{Chi}^{2}$} \\
\hline & & $n^{\circ} \quad \%$ & & $n^{a} \quad(\%)$ & \\
\hline \multicolumn{6}{|l|}{ Sexo } \\
\hline Masculino & 103.511 & $(73,7)$ & 35.172 & $(79,7)$ & 639,42 \\
\hline Femenino & 36.865 & $(26,3)$ & 8.958 & $(20,3)$ & $(p-0,001)$ \\
\hline \multicolumn{6}{|l|}{ Nivel de Estudios } \\
\hline Hasta el 4 año primario & 65.684 & $(49,8)$ & 24.555 & $(55,6)$ & \\
\hline Del $5^{*}$ año al secundario & 34,046 & $(24,2)$ & 8379 & $(18,9)$ & 2555,4 \\
\hline Universitario & 5.923 & $(2,1)$ & 208 & $(0,6)$ & $(p<0,001)$ \\
\hline Sin información & 33.509 & $(23,9)$ & 10.988 & $(24,9)$ & \\
\hline \multicolumn{6}{|l|}{ Categoria de transmisnion } \\
\hline Homosexual & 25.060 & $(17,9)$ & 7.745 & $(17,6)$ & \\
\hline Bisexual & 13.523 & $(9,6)$ & 4.632 & $(10,5)$ & \\
\hline Heterosexual & 34.941 & $(24,9)$ & 9.898 & (22.4) & 31293 \\
\hline Uso de droga inyectable & 23.437 & $(16.7)$ & 11.325 & $(25.7)$ & $(\rho<0.001)$ \\
\hline Otras & 8.491 & $(6,0)$ & 871 & $(2,0)$ & \\
\hline Sin información & 34.924 & $(24,6)$ & 9.659 & $(21,9)$ & \\
\hline \multicolumn{6}{|l|}{ Regioin de residencia } \\
\hline Norte & 2.457 & $(1,8)$ & 820 & $(1,9)$ & \\
\hline Nordeste & 13.017 & $(93)$ & 3.505 & ( 799$)$ & 1361,9 \\
\hline Sureste & 95.144 & $(67,8)$ & 33.674 & $(69,9)$ & $(\rho-0,001)$ \\
\hline Sur & 22,031 & $(15,7)$ & 4.733 & $(10,7)$ & \\
\hline Centro-Qeste & 7.645 & $(5,4)$ & 1.376 & $(3,1)$ & \\
\hline Sin información & 82 & & 22 & & \\
\hline \multicolumn{6}{|l|}{ Periodo de diagenástico } \\
\hline $1980-1986$ & 1.494 & $(1,1)$ & 448 & $(1,0)$ & \\
\hline 1987-1991 & 13.017 & $(18,0)$ & 13.017 & $(20,4)$ & 878.9 \\
\hline $1992-1995$ & 95.144 & $(36,3)$ & 95.144 & (41.9) & $(p=0,001)$ \\
\hline $1996-2000$ & 22.031 & $(44,6)$ & 22.031 & $(36,7)$ & \\
\hline \multicolumn{6}{|l|}{ Edad } \\
\hline (promedio)' & 32.9 & $(11,78)$ & 33,5 & $(8,92)$ & $\begin{array}{c}10.2 \\
(\mathrm{p}-0.001)\end{array}$ \\
\hline
\end{tabular}

1 Utilizada el test $\mathrm{T}$ para igualdad de medias.

de protección. Sin embargo, el análisis ajustado reveló que había mayores prevalencias de tuberculosis, el doble, entre personas que relataron esas categorías de transmisión. Este cambio de dirección en el riesgo sugiere interacción con otros factores.

La tabla 3 presenta datos análogos para casos notificados pertenecientes al sexo femenino. El análisis ajustado por regresión en casos ocurridos entre mujeres comprobó que mayores prevalencias de tuberculosis estaban asociadas significativamente a: uso de drogas inyectables, transmisión heterosexual, baja escolaridad, diagnóstico efectuado antes de 1996, residencia en la región sureste del país y edades menores a 40 años. La categoría de transmisión por medio de relaciones heterosexuales fue comparada con las restantes y se observó en el análisis bivariado que tal categoría conferiría protección. Con todo, el análisis ajustado reveló que esa categoría mostraba asociación con mayores prevalencias de tuberculosis con o sin otras infecciones oportunistas sugiriendo también este cambio la existencia de interacción.

Al comparar los niveles de instrucción en todas categorías de presencia / ausencia de infección oportunista con o sin tuberculosis, según la clasificación en 4 grupos menciona- 
Tabla 2

Análisis bivariado y regresión logística de la asociación entre factores seleccionados y la presencia de tuberculosis sola o acompañada de otras infecciones oportunistas (IO) en casos notificados de sida del sexo masculino. Brasil, 1980-2000

\begin{tabular}{|c|c|c|}
\hline Variables / categoria & $\begin{array}{c}\text { OR bruto } \\
\text { IC95\% }\end{array}$ & $\begin{array}{c}\text { OR ajustudo } \\
\text { IC95\% }\end{array}$ \\
\hline Regiōn de residencia: región Sureste del Brasil & $(1,46-1,54)$ & $1,46(1,41-1,51)$ \\
\hline Estudios: analfabeto y menos de 5 ańos & $(1,67-1,78)$ & $1,54(1,49-1,60)$ \\
\hline Ednd menor que 40 nî̀os & $(1,17-1,24)$ & $1,08(1,04-1,13)$ \\
\hline Diagnostiendo antes de 1996 & $(1,26-1,32)$ & $1,38(1,33-1,43)$ \\
\hline Transmisión: beterosextual & $(0,94-1,00)$ & $2,06(1,85-2,28)$ \\
\hline Transmisión: homo-biscxual & $(0,80-0,85)$ & $1,94(1,75-2,14)$ \\
\hline Transmision: uso de drogas inyectables (JDD) & $(1,52 \cdot 1,62)$ & $2,60)(2,34-2,88)$ \\
\hline
\end{tabular}

1. En el análisis bivariado fueran excluidos $74(0.1 \%)$ casos sin información sobre región de residencia, $1.775(1,3 \%)$ casos sin información sobre edad, $32.212(23,2 \%)$ casos sin información de la infección oportunista y $34.992(25,2 \%)$ casos sin información sobre el nivel de estudio.

2. En la regresión logística fueran excluidos $55.405(40,0 \%)$ casos del análisis.

Tabla 3

Análisis bivariado y regresión logística de la asociación entre factores seleccionados y la presencia de tuberculosis sola o acompañada de otras infecciones oportunistas (IO) en casos notificados de sida del sexo femenino. Brasil, 1980-2000

\begin{tabular}{|lcc|}
\hline Variables / categoria & OR bruto IC95\% & OR ajustado IC95\% \\
\hline Región de residencia: región Sureste del Brasil & $1,63(1,54-1,72)$ & $1,56(1,45-1,67)$ \\
Estudios anal fabeto y menos de 5 años & $2,28(2,13-2,45)$ & $1,72(1,58-1,87)$ \\
Edad: menor que 40 aìos & $1,21(1,14-1,28)$ & $1,12(1,04-1,22)$ \\
Diagnosticado antes de 1996 & $1,57(1,50-1,65)$ & $1,62(1,52-1,73)$ \\
Transmisión: heterosexual & $0,81(0,77-0,86)$ & $2,24(1,92-2,60)$ \\
Transmisión: uso de drogas inyectables (UDI) & $1,21(1,14-1,28)$ & $3,71(3,16-4,36)$ \\
\hline
\end{tabular}

1. En el análisis bivariado fueron excluidos $30(0.1 \%)$ casos sin información sobre región de residencia, $1.358(3,0 \%)$ casos sin información sobre edad, $12.371(23,2 \%)$ casos sin información de la infección oportunista y $10.719(23,4 \%)$ casos sin información sobre el nivel de estudio.

2. En la regresión logística fueran excluidos $19.416(42,4 \%)$ casos del análisis.

da anteriormente (tabla 4), hay una predominancia de individuos cuya categoría de transmisión fue la relación heterosexual y de aquellos que se infectaron por medio de inyecciones de substancias en el grupo de menor escolaridad. Entre individuos de mayor grado de instrucción, notase que la categoría de transmisión predominante en todos los grupos de infección oportunista es la de relaciones homo / bisexuales. Aunque el número de casos cuya categoría de transmisión es UDI sea comparativamente

Rev Esp Salud Pública 2003, Vol. 77, N. ${ }^{\circ} 5$ 
Tabla 4

Distribución del grado de instrucción, categorías de transmisión y la presencia de tuberculosis (TB) u otras infecciones oportunistas (IO) en casos notificados de sida. Brasil, 1980-2000

\begin{tabular}{|c|c|c|c|c|c|c|c|c|c|}
\hline \multirow{2}{*}{$\begin{array}{c}\text { Grado de } \\
\text { Instrucción }{ }^{1}\end{array}$} & \multirow{2}{*}{$\begin{array}{l}\text { Categoria de } \\
\text { transmisión }\end{array}$} & \multicolumn{2}{|c|}{ Sin IO vs. otres } & \multicolumn{2}{|c|}{$\begin{array}{c}\text { Sólo TB vs. } \\
\text { otros }\end{array}$} & \multicolumn{2}{|c|}{$\begin{array}{c}\text { Otras IO ve } \\
\text { otros }\end{array}$} & \multicolumn{2}{|c|}{$\begin{array}{c}\text { TBtotras IO vs. } \\
\text { otros }\end{array}$} \\
\hline & & $n^{*}$ & $(*)$ & $n^{\circ}$ & $(\%)$ & $n^{\circ}$ & $(\bullet)$ & $n^{\circ}$ & $(*)$ \\
\hline \multirow[t]{4}{*}{ Mavor } & Homo bisexual & 3.411 & $(39,7)$ & 723 & $(52,5)$ & 12.907 & $(53,2)$ & 3.386 & $(57,8)$ \\
\hline & Heterosexual & 2.111 & $(24,6)$ & 354 & $(25,7)$ & 5.522 & $(22,8)$ & 1285 & $(21,9)$ \\
\hline & UDI & 617 & $(7,2)$ & 244 & $(17,7)$ & 2.523 & $(10,4)$ & 1.006 & $(17,2)$ \\
\hline & $\mathrm{Otms}^{4}$ & 2.448 & $(28,5)$ & 55 & $(4,0)$ & 3.293 & $(13,6)$ & 179 & $(3,1)$ \\
\hline \multirow[t]{4}{*}{ Mener ${ }^{3}$} & Homo bisexual & 3.270 & $(25,1)$ & 1.183 & $(24,6)$ & 10.851 & $(28,0)$ & 4.457 & $(28,9)$ \\
\hline & Heterosexual & 6.296 & $(48,3)$ & 1.726 & $(35,9)$ & 15.122 & $(39,0)$ & 4.908 & $(31,1)$ \\
\hline & UDI & 3.082 & $(23,6)$ & 1.802 & $(37,5)$ & 11.511 & $(29,7)$ & 5.828 & $(37,8)$ \\
\hline & Otras ${ }^{4}$ & 387 & $(3,0)$ & 100 & $(2,1)$ & 1.241 & $(3,2)$ & 344 & $(2,2)$ \\
\hline
\end{tabular}

. Excluidos 72.434 (39,3\%) casos sin información sobre el nivel de estudio, categoría de transmisión e infección oportunista. . Mayor grado de instrucción: más del $5^{\circ}$ año primario.

. Menor grado de instrucción: hasta el $4^{\circ}$ año primario.

4. Otras categorías de transmisión: por transfusión, transmisión vertical y asociada a hemofilia.

pequeño en estos individuos con mayor instrucción, la participación proporcional de esta categoría de transmisión es mayor en los dos grupos en que la tuberculosis está presente (cerca de 17\%).

Al analizar en detalle los casos con menores niveles de instrucción, entre individuos de sexo masculino de los grupos de infección oportunista en que está presente la tuberculosis, la categoría de transmisión predominante es la debida a UDI (39\%). En contrate, entre los otros dos grupos ( $\sin$ infección oportunista y presencia de otras infecciones oportunistas), la categoría predominante fue la que tuvo transmisión por relaciones homosexuales (cerca de $38 \%$ ), en el sexo masculino, y heterosexuales (78\%), en el sexo femenino.

Fueron comparadas las 4 categorías de exposición (homo/bisexual, heterosexual, UDI y las restantes) dentro de cada uno de los grupos de escolaridad. Al someter a prueba las diferencias encontradas, los resultados demuestran que existen diferencias estadísticamente significativas en la distri- bución de las categorías de infección oportunista en cada grupo de escolaridad (para el grupo de mayor escolaridad: Pearson $X^{2}$ $2.446,4$, con 9 GL, $\mathrm{p}<0,01$; para el grupo de menor escolaridad: Pearson $X^{2} 1.193,8 ; 9$ GL, $\mathrm{p}<0,01)$.

La tabla 5 muestra las tendencias de la evolución de la presencia de tuberculosis en los casos notificados demostrándose un aumento inicial de $23 \%$ para $26 \%$, que se mantuvo en los periodos siguientes. Sin embargo, después de 1996, tal tendencia se anula llegando a $20 \%$ la proporción de casos con tuberculosis. Al estudiar las mismas tendencias discriminando los 4 grupos según la presencia o ausencia de enfermedad oportunista y la presencia de tuberculosis aislada o asociada a esas enfermedades (datos no mostrados en tablas), fue demostrado un aumento significativo de la proporción de pacientes sin enfermedades oportunistas y de pacientes solamente con tuberculosis en cuatro períodos consecutivos. Fueron observados incrementos de $116 \%$ y de $20 \%$ en el número de personas no portadoras de infec- 
Tabla 5

Análisis de tendencia linear de la asociación entre factores seleccionados y la presencia de tuberculosis (TB) en casos notificados de sida en cuatro períodos. Brasil, 1980-2000

\begin{tabular}{|c|c|c|c|}
\hline Periodos & $\operatorname{Sin} \mathrm{TB}^{\prime}$ & $\operatorname{Con} \mathrm{TB}^{1}$ & $\mathrm{Chi}^{2} /$ valor $\mathrm{p}^{2}$ \\
\hline $1980-1986$ & $1.494 \quad(76,9 \%)$ & $448 \quad(23,1 \%)$ & 579,46 \\
\hline $1987-1991$ & $25.240 \quad(73,7 \%)$ & $8.988 \quad(26,3 \%)$ & $\mathrm{p}<1 \times 10^{-4}$ \\
\hline $1992-1995$ & $50.990 \quad\left(73,4^{\circ} \%\right)$ & $18.502(26,6 \%)$ & \\
\hline $1996-2000$ & $62.652 \quad(79.5 \%)$ & $44.130(20.5 *)$ & \\
\hline
\end{tabular}

Número de casos válidos computados $=184.506$

Utilizada la prueba Chi cuadrado para tendencia linear.

ciones oportunistas y portadoras de tuberculosis sin otras infecciones oportunistas entre los periodos 1996-2000 y 1992-1995, respectivamente. En esos mismos periodos se observó una disminución de $23 \%$ en la proporción de pacientes portadores de tuberculosis acompañada de otras infecciones oportunistas. Estos cambios fueron todos significativos $\left(\mathrm{Chi}^{2}\right.$ oscilando entre 126,6 e 3.400 ; valores $\mathrm{p}<1 \times 10^{-4}$ ).

\section{DISCUSIÓN}

Existe una proporción mayor de varones en todas las categorías analizadas. Sin embargo, esta predominancia es más acentuada, cerca de $80 \%$, en presencia de tuberculosis. Esta sobre-representación del sexo masculino es debida no a los riesgos diferenciados según el sexo sino a los efectos de la razón de notificación hombre: mujer que, apesar del declinio a lo largo de los anõs 80 y 90, continúa reflejando la ocurrencia en un número mayor de hombres, siendo actualmente de 2:1, aproximadamente. Es posible, sin embargo, que la ausencia de algunas informaciones en determinadas variables, cuyo peso cuantitativo está resaltado en las notas al pié de las tablas, haya contribuído para introducir sesgos que magnifican falsamente esa sobre-representación.
Con respecto a la edad, nuestro estudio mostró que los promedios son mayores en las categorías en que la tuberculosis está presente. Sin embargo, al dicotomizar la variable, los menores de 40 años tienen un riesgo mayor de presentar tuberculosis sola o asociada a otras infecciones oportunistas. Tal efecto no se mantuvo en el análisis multivariado, para ambos sexos, debido a presencia de otras variables en el modelo con mayor capacidad discriminatoria para la predicción de la tuberculosis entre los casos notificados.

Los casos con baja escolaridad predominaban en las categorías en que la tuberculosis estaba presente sola o con otras infecciones oportunistas, variable que también permaneció asociada después de ajustar los estimadores para la presencia de otras variables. Aunque no se disponga de informaciones sobre el salario promedio mensual o acerca del tipo de ocupación de los casos notificados, los datos sugieren que los casos de tuberculosis y sida pueden pertenecer a grupos sociales menos privilegiados e por tanto, presentar mayor riesgo de exposición al $M$. tuberculosis. Estudios sobre las tendencias epidemiológicas del sida en el Brasil, han demostrado que los casos incidentes han sido cada vez más pobres en el transcurso de las dos décadas de la epidemia ${ }^{36}$. Es justamente entre esas capas menos privilegiadas de la sociedad, ahora alcanzadas por el sida, 
donde historicamente ha ocurrido predominantemente la tuberculosis. Los niveles de escolaridad observados son consistentes con la tendencia ya observada de los casos notificados de sida a reflejar progresivamente las condiciones generales de escolaridad en el país. Según datos del Instituto Brasileño de Geografía y Estadística (IBGE) $)^{37}$, la proporción de analfabetismo en la población se redujo de $14 \%$ a $11 \%$ al final de la década de 1990. Sin embargo, solamente $37 \%$ de la población ha alcanzado más del nivel primario completo que corresponde a 8 años de enseñanza.

La forma de transmisión predominante fué la heterosexual, entre los casos con información. No obstante, la forma más relacionada con la presencia de tuberculosis fué la utilización de drogas inyectables. Estos hallazgos están en concordancia con estudios que señalan una interacción compleja entre el uso de drogas, prácticas sexuales de riesgo, bajo nivel socio-económico, presencia de infección por el virus HIV o de sida y el riesgo de desarrollar la tuberculosis ${ }^{13,38}$. El análisis ajustado terminó mostrando asociación entre la presencia de tuberculosis y la via de transmisión homosexual a pesar de no ser la asociación más fuerte entre los distintos mecanismos de contagio. Otros estudios encuentran una asociación entre prácticas exclusivamente homosexuales y la presencia de tuberculosis ${ }^{24}$, pero la relativa debilidad en la magnitud de la asociación podría ser explicada en parte por la diferencia en la distribución por nivel educacional. En nuestro estudio fue observado que individuos que cursaram hasta el $4^{\circ}$ año primario y que reportaron prácticas de uso de drogas inyectables estuvieron claramente sobrerepresentados cuando la infección oportunista incluía la tuberculosis.

Con respecto al periodo de diagnóstico, observamos que hubo un aumento en los casos notificados sin infección oportunista en el último periodo (1996 a 2000) comparado a los anteriores debido, muy probable- mente, a un cambio en la definición de caso que incluyó a los individuos con serología positiva para el VIH y conteo de CD4 menor de 500. Sin embargo, en los dos períodos (1992-1995 y 1996-2000) hubo una estabilización en la proporción de casos solamente con tuberculosis y una disminución de los que presentaban tuberculosis asociada a otras infecciones oportunistas. Esto se debe, probablemente, a la distribución gratuita desde 1996, por parte del gobierno brasileño, de los inhibidores de la proteasa en la lista de medicamentos para el tratamiento del $\operatorname{sida}^{29}$, disminuyendo la incidencia de las infecciones oportunistas y aumentando la supervivencia de los pacientes ${ }^{39}$. Hay evidencias de una reducción en la mortalidad por sida atribuible a este hecho ${ }^{40}$. Sin embargo, aún donde hay disponibilidad de la terapia HAART, la disminución de la incidencia de la tuberculosis está relacionada también con factores que influyen en la adesión al tratamiento de AIDS, tales como la calidad de los servicios de salud y las condiciones de vida de los pacientes. Los servicios que atienden los pacientes con tuberculosis aún carecen de infraestructura que les permita ofrecer la prueba diagnóstica del VIH, lo que detectaría precozmente los casos coinfectados instituyendo oportunamente un tratamiento supervisado adecuado. Además, es plausible que el aumento de la desigualdad social y económica en el Brasil en las últimas dos décadas ${ }^{41}$, haya contribuído para que un número cresciente de personas estén sometidas a condiciones precarias de vida, con el consecuente mayor riesgo de infección por el VIH y de desarollar la tuberculosis.

A pesar de que los datos de nuestro estudio señalen un aumento de la proporción de casos cuya forma de transmisión fueron las relaciones heterosexuales en los casos notificados, debemos resaltar que el porcentaje de casos que relataron prácticas homosexuales no necesariamente refleja la tasa de incidencia. Por lo tanto, no se puede afirmar categóricamente si hay un aumento real de la 
transmisión heterosexual o si se trata de un aumento relativo en virtud de una disminución de la tasa de incidencia entre individuos con prácticas homo y bisexuales. Aunque no se pretenda negar la posibilidad de un verdadero aumento de casos de transmisión heterosexual, no se puede descartar tampoco la posibilidad de errores de clasificación de la categoría de exposición.

Vale la pena destacar que por tratarse de un estudio con datos secundarios originados en las fichas de notificación de casos de sida, el atraso o la subnotificación pueden haber afectado los resultados del estudio. La presencia de sesgos en la selección en este estudio puede ser consecuencia de la unidad de salud que realiza la notificación. Los casos de sida atendidos en consultorios y/o hospitales particulares puede estar sub-representado en el universo de casos notificados. No tenemos datos sobre la proporción de pacientes diagnosticados con sida que son tratados en los servicios privados del país. Sin embargo, debido al alto costo del tratamiento antiretroviral, podemos suponer que es escaso, principalmente sabiendo que los individuos infectados en los últimos años han sido progresivamente más pobres. La Investigación Nacional de Muestreo por Domicilios, efectuada por el IBGE en $1998^{42}$, mostró que la proporción general de uso de servicios públicos era de cerca de $50 \%$ y que solamente $25 \%$ de las personas estaban cubiertos por algún seguro de salud.

Los individuos detectados en hospitales universitarios y entidades públicas de referencia para tuberculosis y sida tienen mayores probabilidades de ser confirmados, dada la presencia de profesionales más actualizados en relación a la asociación de tuberculosis - sida y de mayor oferta de medios de diagnóstico, con respecto a individuos notificados por centros de salud o hospitales estatales, lo que constituye sesgo de detección. Aunque la confirmación de un caso de tuberculosis no necesite recursos diagnósticos sofisticados, el diagnóstico diferencial de micobacteriosis atípicas, o de otras patologías pulmonares, requiere personal técnico especializado e infraestructura de laboratorio con cierto nivel de complejidad. Tal hecho podría estar influyendo en la clasificación correcta de los casos de tuberculosis en las distintas unidades que notifican. Además de eso, por no disponer de las fechas de diagnóstico tanto de la tuberculosis como del sida, no fue posible evaluar, en nuestro medio, si el diagnóstico de la primera precedía el del segundo.

Nuestro estudio no llega a especificar la proporción de pacientes portadores de micobacterias atípicas. Según Hijjar y colaboradores $(2001)^{43}$, mientras $30 \%$ de los casos notificados de sida en el Brasil presentan tuberculosis en todas las formas, solamente $2,5 \%$ tienen manifestaciones clínicas de micobacterias atípicas. Un estudio documenta la distribución de los resultados de exámenes enviados al laboratorio nacional de referencia, en Rio de Janeiro, por sospecha de micobacteriosis atípica -590 cultivos en 6 años- habiendo sido verificada y tipificada la presencia de tales microorganismos ${ }^{44}$. Estos resultados pueden estar reflejando más la realidad de casos de tuberculosis extra-pulmonar o de fallas terapéuticas. En ese mismo período, de 1994 a 1999, el promedio de casos de tuberculosis notificados fue de cerca de 90.000 por año, razón por la cual se puede suponer que la micobacteriosis atípica no es tan frecuente como en otros medios. Sin embargo, no han sido realizados estudios sistemáticos ni en los pacientes ni en ámbito de población que determinen la frecuencia real de su ocurrencia en el país.

Ante la falta de orientaciones técnicas que conduzcan a la sospecha de infección por el $\mathrm{VIH}$ en pacientes con tuberculosis, nuestro objetivo en este estudo fue destacar las variables indicadoras de la presencia de tuberculosis en los casos de sida, auxiliando a los profesionales que trabajan en los servicios de salud en la sospecha de la coinfec- 
ción. En el Brasil, las acciones dirigidas al control de la coinfección por la tuberculosis y sida reflejan las características de la estructura organizacional y de los recursos financieros y humanos disponibles. El ofrecimiento de la prueba diagnóstica de VIH para la detección precoz de la coinfección en los pacientes con tuberculosis depende de otras cuestiones concernientes a la reorganización general de los servicios de salud que hoy se encuentran en marcha en el Brasil, como la descentralización de las actividades de control de tuberculosis y de sida por medio del programa de atención básica en salud que aún necesita de normatización técnica y operacional por parte del nivel federal de gobierno. De esto dependen otras cuestiones relevantes para este tema como el aconsejamiento pre y post-diagnóstico, y la expansión del tratamiento supervisado para tuberculosis y para sida. Todos estos problemas pueden estar englobados en lo que Jonathan Mann llamó «vulnerabilidad programática» ${ }^{45}$.

Una respuesta efectiva al problema de la coinfección debe tener en cuenta la particularidad de los medios de transmisión de esas enfermedades y del fuerte componente estigmatizante asociado a ellas. Esperaríamos un impacto positivo en el cuadro epidemiológico de las dos enfermedades con la adopción de medidas ya conocidas para el control de la tuberculosis: sospecha de tuberculosis en individuos sintomáticos respiratorios, diagnóstico precoz, esquemas terapéuticos adecuados, garantía de adesión al tratamiento y investigación de los comunicantes. Otra medida de impacto sería la garantía de acceso a los medios de diagnóstico de HIV y al tratamiento antiretroviral. No obstante, como subraya Selwyn ${ }^{4}$, «esperemos que los abordajes paradigmáticos a estas dos epidemias sean complementarios y no competidores, de tal modo que las estrategias requeridas para controlar cualquiera de ellas no irán a comprometer los esfuerzos dirigidos a la otra». Adicionalmente, en un contexto de políticas económicas recesivas, desmantelamiento de los servicios públicos y aumento de la atención a la salud por parte del sector privado, es necesario que las acciones de los programas de control de la tuberculosis y del sida estén asociadas con estrategias de reducción de la desigualdad social, para que se logre un éxito terapéutico con relevancia epidemiológica en el control de estas patologías.

\section{BIBLIOGRAFÍA}

1. Lienhardt C, Rodrigues LC. Estimation of the impact of the human immunodeficiency virus infection on tuberculosis: tuberculosis risks re-visited? Int J Tuberc Lung Dis 1997; 1:196-204.

2. Mayaud C, Cadranel J. Tuberculosis in AIDS: past or new problems? Thorax 1999;54:567-71.

3. Centers for Disease Control and Prevention (CDC). Tuberculosis and Acquired Immunodeficiency Syndrome : New York City. MMWR 1987; 36:785-7.

4. Selwyn PA. Tuberculosis in the AIDS era: a new threat from an old disease. New York State J Med 1991; 91:233-5.

5. Ministerio de Salud (MS). Coordinación Nacional de DST/sida. Revisión de la definición de caso de sida en adultos. Brasil: MS, 1992.

6. Castilho EA, Chequer P. Epidemiologia do HIV/AIDS no Brasil. En: Parker R. organizador. Políticas, Instituições e AIDS. Enfrentando a Epidemia no Brasil. 1a ed. Rio de Janeiro: Jorge Zahar Editor/ABIA; 1998. p. 17-42.

7. Narain JP, Raviglione MC, Kochi A. HIV-associated tuberculosis in developing countries: epidemiology and strategies for prevention. Tubercle Lung Dis 1992; 73:311-21.

8. Nunn P, Gicheha C, Hayes AM. Cross-sectional survey of HIV infection among patients with tuberculosis in Nairobi, Kenya. Tubercle Lung Dis 1992; 73:45-51.

9. García MLG, Gómez JLV, Sancho MCG, Álvarez RAS, Zacarías F, Amor JS. Epidemiología del sida y la tuberculosis. Bol Oficina Sanit Panam 1994;116:546-65.

10. Hypollite PR, Pape JW. Infección por HIV y tuberculosis en Haití. Bol Oficina Sanit Panam 1995; 118:161-9. 
11. Broek JVD, Borgdorff MW, Pakker NG, Chum HJ, Klokke AH, Senkoro KP et al. HIV-1 Infection as a risk factor for the development of tuberculosis: a case-control study in Tanzania. Int J Epidemiol 1993; 22:1159-65.

12. Selwyn PA, Hartel D, Lewis, Schoenbaum EE, Vermund SH, Klein RS et al.. A prospective study of the risk of tuberculosis among intravenous drug users with human immunodeficiency virus infection. N Engl J Med 1989: 320:545-50.

13. Cayla JA, García de Olalla PG, Galdós-Tanguis H, Vidal R, López-Colomés JL, Gatell JM et al. The influence of intravenous drug use and HIV infection in the transmission of tuberculosis. AIDS 1996; 10:95-100

14. Del Amo J, Petruckevitch A, Phillips AN, De Cock KM, Stephenson J, Desmond N et al. Risk factors for tuberculosis in patients with AIDS in London: a case-control study. Int J Tuberc Lung Dis , 1999; $3: 12-7$.

15. Rieder HL. Epidemiologic basis of tuberculosis control. Paris : International Union Against Tuberculosis and Lung Disease ; 1999.

16. Colenbunders R, Lambert ML. Management of coinfection with HIVand TB. BMJ 2002 ;324 :802-3.

17. Ministerio de Salud del Brasil (MS). Coordinación Nacional de DST / AIDS. Aids: Boletim Epidemiólogico - AIDS 2001 ; XIV (2):7.

18. Ruffino Neto A. Avaliação do excesso de casos de tuberculose atribuídos a infecção pelo HIV/AIDS: ensaio preliminar. Rev Saúde Pública 1995; 29:279-82.

19. Kritski AL, Werneck EB, Vieira MA, Carvalho CCA, Carvalho CE, Bravo de Souza R et al. HIV infection in 567 Active Pulmonary Tuberculosis patients in Brasil. J Acquired Immune Deficiency Syndromes $1993 ; 6: 1008-12$.

20. Kritski AL, Dalcomo M, del Bianco R, Melo FF, Pinto WP, Schechter M et al. Associação tuberculose e infecção pelo HIV no Brasil. Bull Pan-American Health Office $1995 ; 11: 542-554$.

21. Kritski AL, Lapa e Silva JR, Conde MB. Tuberculosis and HIV: Renewed Challenge. Mem Inst Oswaldo Cruz 1998; 93:417-421.

22. Toledo AS. Indicadores de rastreamento para detecção de infecção pelo HIV entre tuberculosos. Bol Pneumol Sanit 1997; 5:43-9.

23. Kritski AL, Werneck EB, Silva RA. Association between tuberculosis and AIDS in 135 patients. Am Rev Respir Dis 1989; 139: A147.
24. Kerr-Pontes LRS, Oliveira FAS, Freire CAM Tuberculose associada à AIDS: situação de região do Nordeste brasileiro. Rev Saúde Pública 1997; 31:323-329.

25. Osawa CR, Soares TB, Peixoto SB, Cassi RM, de Riemer K. Tuberculosis among AIDS patients in Santos, Brasil, 1987-1996. En : EPI-RIO 98. Livro de Resumos do IV Congresso Brasileiro de Epidemiologia ; 1998 agosto 1-5 ; Riuo de Janeiro, Brasil. Rio de Janeiro : ABRASCO ; 1998. p. 178

26. Santo, AH, Pinheiro CE, Jordani MS. Causas básicas e associadas de morte por AIDS. Estado de São Paulo, Brasil, 1998. Rev Saúde Pública 2000; 34:581-8.

27. Ministerio e Salud del Brasil. (MS). Informe técnico - Mímeo. Área Técnica de Pneumologia Sanitária (ATPS) / Secretaría de Políticas de Salud (SPS). Brasilia : Ministerio de Salud, 2000.

28. [Referência a ser entrada aqui]

29. Galvão J. Access to antiretroviral drugs in Brazil. Lancet 2002; 360 (9348) : 1862-65

30. Lima MM, Belluomini M, Almeida MMMB, Arantes GR. Co-infecção HIV-Tuberculose: necessidade de uma vigilância mais efetiva. Rev Saúde Pública 1997; 31:217-20.

31. Antunes JLF, Waldman EA . The impact of AIDS, immigration and housing overcrowding on tuberculosis deaths in São Paulo, Brazil - 1994-1998. Soc Sci Med 2001; 52 :1071-80.

32. Knigge A, Morr H, Kilian A . Tuberculosis and HIV/AIDS epidmeics : opportunities for cross support. $1^{\mathrm{a}}$ ed. Eschborn: Deutsche Gesellschaft für Technische Zusammenarbeit, 2000.

33. Frieden TR. Can tuberculosis be controlled ? Int J Epidemiol 2002; 31:894-99.

34. Epi Info. Procesador de texto, base de datos y estadística para epidemiología. Programa de ordenador de dominio público. Versión 6-04. Atlanta (GA, USA) : USDHHS/CDC ; 1995.

35. SPSS. Programa de ordenador adquirido por la CNDST/AIDS (Ministério da Saúde do Brasil). Versión 9.0. Chicago (IL, USA) : SPSS Inc. 1999.

36. Szwarcwald CL, Bastos FI, Castilho EA. The dynamics of the AIDS epidemic in Brazil: A space-time analysis in the period 1987-1995. Brazilian J Infec Dis 1998; 2: 175-186.

Rev Esp Salud Pública 2003, Vol. 77, N. ${ }^{\circ} 5$ 
37. IBGE http://www.ibge.gov.br/brasil_em_sintese/ default.htm.

38. Schoenbaum EE, Hartel D, Selwyn, PA, Klein RS, Davenny K, Rogers M et al. Risk Factors for Human Immunodeficiency Virus infection in intravenous drug users. N Engl J Med 1989; 321: 87479.

39. Marins JRP, Jamalw LF, Chen S, Hudes ES, Barbosa Jr. A, Barros MBA et al. Sobrevivência atual dos pacientes com AIDS no Brasil : evidência dos resultados de um esforço nacional. Boletim Epidemiológico - AIDS 2002 ; XV (1): 37-47.

40. Fonseca MGP, Barreira D. A evolução da mortalidade por AIDS no país, segundo sua distribuição geográfica. Boletim Epidemiológico - AIDS 2000 ; XIII (3): 43-49.

41. Minayo MCS. Condiciones de vida, desigualdad y salud a partir del caso brasileño. En: Briceño-León
R, Minayo MCS, Coimbra Jr. CEA (coordinadores). Salud y Equidad: una mirada desde las ciencias sociales. $1^{\mathrm{a}}$. Edición. Rio de Janeiro: Editora FIOCRUZ; 2000. p. 55-71.

42. IBGE. Pesquisa Nacional por Amostra de Domicílios (PNAD). 1998.

43. Hijjar MA, Oliveira MJPR, Teixeira GM. A tuberculose no Brasil e no mundo. Boletim de Pneumologia Sanitária 2001; 9: 9-16.

44. Barreto AMW, Campos CED. Micobactérias «não tuberculosas» no Brasil. Boletim de Pneumologia Sanitária 2000; 8: 23-32.

45. Mann J, Tarantola DJM. From epidemiology to vulnerability, to human rights. En : Mann J, Tarantola DJM. editores. AIDS in the World II. Global Dimensions, Social Roots and Responses. $1^{\mathrm{a}}$ ed. New York : Oxford University Press, 1996. p427462. 